Strahlenther Onkol 2013 · 189:708-708

DOI 10.1007/s00066-013-0374-6

Online publiziert: 25 . Juli 2013

๑) Springer-Verlag Berlin Heidelberg 2013

J. Debus

RadioOnkologie und Strahlentherapie, Universitätsklinikum Heidelberg

\title{
Professor Dr. Dr. Michael Wannenmacher zum 75. Geburtstag
}

lignen Lymphomen erlangt. Diese Arbeiten waren vor allem durch Forschungsaufenthalte bei Gilbert Fletcher in Houston geprägt worden. Seit dieser Zeit hat er die internationale Zusammenarbeit stark gefördert. Er hat die Deutschen HodgkinStudien im Erwachsenen- und Kindesalter seit 1977 wesentlich mitgestaltet und geprägt.

Seit 1977 hatte er den Lehrstuhl für Strahlentherapie in Freiburg inne, nachdem er den Ruf nach Erlangen und später auch nach Hamburg abgelehnt hatte. 1988 wechselte er auf den Lehrstuhl nach Heidelberg. Dort prägte er als leitender Ärztlicher Direktor, Prodekan der medizinischen Fakultät und Sprecher des Tumorzentrums den Standort ganz wesentlich durch seine Person. Als Präsident der Deutschen Krebsgesellschaft hat er nachhaltig die onkologischen Strukturen in Deutschland mitgestaltet und die Krebsgesellschaft in schwierigen Zeiten stabilisiert. Er gab dem Deutschen Krebskongress 1994 ein eigenes Format, indem er besonders die Interdisziplinarität herausstellte. Insgesamt hat Michael Wannenmacher die multidisziplinäre Zusammenarbeit nicht nur gefordert, sondern auch intensiv vorgelebt. Die Heidelberger Zeit war wesentlich geprägt durch die wissenschaftliche Entwicklung von stereotaktischen Radiotherapieverfahren und den Aufbau des Deutschen Schwerionentherapie-Projektes. Mit diesen Technologien hat Michael Wannenmacher international hohes Ansehen erlangt. Seine Patienten schätzten sein freundliches Wesen und die vertrauensvolle Art. Bezeichnend ist, dass in der deutschen Radioonkologie acht Lehrstühle und viele Chefarztpositio- nen mit seinen ehemaligen Mitarbeitern besetzt wurden.

Seine Arbeit und seine Verdienste um die Onkologie wurden durch zahlreiche Ehrungen und Preise ausgezeichnet, darunter das Bundesverdienstkreuz, die Röntgen-Medaille sowie die K.H.-BauerMedaille.

Seit seiner Emeritierung ist Michael Wannenmacher mit dem Aufbau eines Comprehensive Cancer Centers in Doha/ Qatar beschäftigt und als Berater bei der Dietmar-Hopp-Stiftung aktiv. Er ist leidenschaftlicher Golfspieler und verbringt viel Zeit mit Reisen und seiner Familie. Besonderen Stolz bereiten ihm seine beiden Enkel in Hongkong.

Prof. Wannenmacher steht seinen Schülern stets mit der ihm eigenen freundlichen Zurückhaltung mit Rat und Tat zur Seite. Dadurch prägt er mit großem Wissensschatz und viel Erfahrung auch heute noch über seine Schülerinnen und Schüler die radioonkologische Landschaft. Wir wünschen ihm weiterhin viel Freude an den Früchten seines Erfolges und gesundheitlich alles Gute.

Jürgen Debus, Heidelberg

\section{Korrespondenzadresse}

Prof. Dr. Dr. J. Debus

RadioOnkologie und Strahlentherapie, Universitätsklinikum Heidelberg Heidelberg

Juergen.Debus@med.uni-heidelberg.de

Interessenkonflikt. Der korrespondierende Autor gibt an, dass kein Interessenkonflikt besteht. 